MATHEMATICS OF COMPUTATION

Volume 71, Number 239, Pages 1021-1042

S 0025-5718(01)01340-0

Article electronically published on October 26, 2001

\title{
NUMERICAL COMPUTATION OF VISCOUS PROFILES FOR HYPERBOLIC CONSERVATION LAWS
}

\author{
HEINRICH FREISTÜHLER AND CHRISTIAN ROHDE
}

\begin{abstract}
Viscous profiles of shock waves in systems of conservation laws can be viewed as heteroclinic orbits in associated systems of ordinary differential equations (ODE). In the case of overcompressive shock waves, these orbits occur in multi-parameter families. We propose a numerical method to compute families of heteroclinic orbits in general systems of ODE. The key point is a special parameterization of the heteroclinic manifold which can be understood as a generalized phase condition; in the case of shock profiles, this phase condition has a natural interpretation regarding their stability. We prove that our method converges and present numerical results for several systems of conservation laws. These examples include traveling waves for the Navier-Stokes equations for compressible viscous, heat-conductive fluids and for the magnetohydrodynamics equations for viscous, heat-conductive, electrically resistive fluids that correspond to shock wave solutions of the associated ideal models, i.e., the Euler, resp. Lundquist, equations.
\end{abstract}

\section{INTRODUCTION AND OUTLINE}

Consider a nonlinear system of conservation laws in one space dimension,

$$
u_{t}+f(u)_{x}=0,
$$

where $x \in \mathbf{R}, t \geq 0, u(x, t)$ takes values in $\mathbf{R}^{m}$, and the flux function $f$ is a smooth nonlinear mapping from (a subset of) $\mathbf{R}^{m}$ to $\mathbf{R}^{m}$. Assume that the (1.1) is hyperbolic; i.e., for each $u$, the Jacobian $D f: \mathbf{R}^{m} \rightarrow \mathbf{R}^{m \times m}$ has $m$ real eigenvalues $\lambda_{1}(u), \ldots, \lambda_{m}(u)$ with

$$
\lambda_{1}(u) \leq \lambda_{2}(u) \leq \cdots \leq \lambda_{m}(u),
$$

and a complete set of associated eigenvectors $r_{1}(u), \ldots, r_{m}(u)$. We are interested in viscous profiles for shock wave solutions of (1.1).

To be specific, for given base states $u^{-}, u^{+} \in \mathbf{R}^{m}$ and shock speed $s \in \mathbf{R}$ a function $U$ with

$$
U(x, t)=\left\{\begin{array}{lll}
u^{-} & : & x<s t \\
u^{+} & : & x>s t
\end{array}\right.
$$

Received by the editor December 16, 1998 and, in revised form, September 12, 2000.

2000 Mathematics Subject Classification. Primary 65L10; Secondary 35L65, 34C37, 76W05.

Key words and phrases. Shock waves, connecting heteroclinic manifolds, boundary value problems for ODE, magnetohydrodynamics.

The authors acknowledge support by the DFG Schwerpunktprogramm "Ergodentheorie, Analysis und effiziente Simulation dynamischer Systeme" and by the EU-TMR research network for Hyperbolic Conservation Laws (project \# ERBFMRXCT960033).

(C)2001 American Mathematical Society 
is called a shock wave solution of (1.1) if $U$ is a solution of (1.1) in the distributional sense; this is the case if and only if the Rankine-Hugoniot conditions hold, i.e.,

$$
f\left(u^{+}\right)-s u^{+}=f\left(u^{-}\right)-s u^{-} \equiv q .
$$

To stay away from degenerate cases - even though these can sometimes be interesting - we assume that the shock waves we consider are noncharacteristic on either side, i.e., $\lambda_{j}\left(u^{ \pm}\right) \neq s$ for $j=1, \ldots, m$. Consequently there exist numbers $k^{-}, k^{+} \in$ $\{0, \ldots, m\}$ such that for $j=1, \ldots, m$

$$
\lambda_{m-j+1}\left(u^{-}\right)>s \text { for } j \leq k^{-}, \lambda_{m-j+1}\left(u^{-}\right)<s \text { for } j>k^{-}
$$

and

$$
\lambda_{j}\left(u^{+}\right)<s \text { for } j \leq k^{+}, \lambda_{j}\left(u^{+}\right)>s \text { for } j>k^{+} .
$$

$k^{-}, k^{+}$are the numbers of incoming modes on either side of the shock. Together with (1.1) consider now also an associated family.

$$
u_{t}+f(u)_{x}=\left(\mathcal{D}(u, \delta) u_{x}\right)_{x}, \quad \delta \in \Delta,
$$

of parabolic or hyperbolic-parabolic systems. Systems of hyperbolic conservation laws which arise in continuum physics are naturally equipped with such families. Here $\Delta \subset[0, \infty)^{p}$ with $0 \in \Delta$ denotes the range of the dissipation parameter $\delta$. The smooth viscosity matrix $\mathcal{D}: \mathbf{R}^{m} \times \Delta \rightarrow R^{m \times m}$ is supposed to satisfy $\mathcal{D}(., 0)=0$. A viscous profile for a shock wave (1.3) is a solution $\phi$ of the ODE boundary value problem

$$
\mathcal{D}(\phi, \delta) \dot{\phi}=f(\phi)-s \phi-q, \quad \phi( \pm \infty)=u^{ \pm},
$$

or, in other words, a heteroclinic orbit from $u^{-}$to $u^{+}$. The motivation for considering profiles is that a solution of (1.8) - if it exists and under appropriate conditions on $\mathcal{D}$-provides a regularized counterpart of the shock wave (1.3) to which latter it converges for $\delta$ tending to 0 . This can be viewed as expressing that this shock wave is compatible with the vanishing dissipation limit. For the analytical background concerning viscous profiles in the context of hyperbolic conservation laws we refer, e.g., to the book of Serre ([19], Chapter 7) and the references therein.

To classify different types of shock waves and viscous profiles simultaneously, we assume for a moment that $\mathcal{D} \equiv \delta I d$, with $I d$ the identity matrix of order $m$ and $\delta>0$ a scalar dissipation parameter. In this case (at least), (1.8) takes the standard form of a first-order ODE and the states

$$
u^{ \pm} \text {are hyperbolic rest points of the vector field }
$$

$$
u \mapsto F(u) \equiv \mathcal{D}^{-1}(u)(f(u)-s u-q),
$$

i.e., the Jacobian of $F$ at $u^{ \pm}$has no eigenvalues on the imaginary axis. Denoting the stable and unstable manifolds of $u^{ \pm}$by $\mathcal{M}_{s}\left(u^{ \pm}\right), \mathcal{M}_{u}\left(u^{ \pm}\right)$and the dimensions of these manifolds by $m_{s}^{ \pm}, m_{u}^{ \pm}$, we let

$$
d \equiv m_{u}^{-}+m_{s}^{+}-m
$$

and observe that

$$
m_{u}^{-}=k^{-}, m_{s}^{+}=k^{+}, \text {and } d=k_{u}^{-}+k^{+}-m .
$$

Now if $d=1$ and $\mathcal{M}_{u}\left(u^{-}\right)$intersects $\mathcal{M}_{s}\left(u^{+}\right)$transversally, then the solution of (1.8) consists of a single orbit. Necessarily, $k^{-}+k^{+}=m+1$, which identifies the underlying shock wave solution $U$ as a classical or Laxian shock. For $d<1$, the 
intersection of $\mathcal{M}_{u}\left(u^{-}\right)$and $\mathcal{M}_{s}\left(u^{+}\right)$is not a structurally stable object and $U$, with $k^{-}+k^{+}<m+1$, is called undercompressive. Completing this picture, for $d>1$, the generic intersection of $\mathcal{M}_{u}\left(u^{-}\right)$and $\mathcal{M}_{s}\left(u^{+}\right)$forms a $d$-dimensional heteroclinic manifold connecting $u^{-}$and $u^{+}$; the shock wave $U$, now with $k^{-}+k^{+}>m+1$, is classified to be overcompressive. We note that (1.9) and (1.11) will of course not automatically hold for choices of $\mathcal{D}$ which are different from multiples of the identity matrix $I$. They do hold in many physically interesting cases however, even though in the typical hyperbolic-parabolic case $\mathcal{D}$ is not even of full rank.

Subject of this paper is the presentation, analysis, and application of a numerical algorithm which allows us to compute the viscous profiles of Laxian and, in particular, of overcompressive shock waves. The method will be formulated and its convergence proved for general autonomous systems of ODE, i.e., not be restricted to shock profile equations. Well-established methods are known from the literature for approximating heteroclinic (or homoclinic) connections that consist of exactly one orbit; we refer to [3, 5, 16]. Less can be found for heteroclinic manifolds of dimension bigger than one [2, 5]; the methods presented (without convergence proofs) in these papers do (moreover) not seem to be conveniently applicable to the case of families of shock profiles as regards their respective suggested ways of singling out the individual orbits in a higher-dimensional heteroclinic manifold. Motivated by results on the time-asymptotic stability of viscous profiles [14, 6], we introduce here a special parameterization for heteroclinic manifolds which does fulfill this necessity. The idea of this parameterization derives from considerations on the time-asymptotic stability of viscous profiles as solutions of the partial differential equations. The parameterization can formally be viewed as evaluating a novel version of what Beyn calls a phase condition [3]. Based on the parameterization, we formulate the announced numerical method.

The remainder of the paper is arranged as follows. Section 2 deals with introducing the novel parameterization. Furthermore a boundary value problem will be presented the solutions of which are single trajectories with fixed end states. In Section 3 we introduce an associated problem on a finite interval, to which end we prescribe asymptotic boundary conditions at its boundary points $X_{ \pm}$. These ingredients yield the numerical method for approximating the heteroclinic manifold. It will be shown that the error induced by the truncation procedure vanishes for $X_{ \pm}$ tending to $\pm \infty$. This main result (Theorem 4.1) is proved in Section 4 the proof follows 3 closely, though with some nontrivial modifications. Finally, in Section 5 we will apply the method first to some instructive simple models, then to physically relevant systems of conservation laws like those of fluid dynamics and those of magnetohydrodynamics. The main emphasis lies on the latter, an example where various different kind of overcompressive shocks waves arise. Note that analytical findings regarding the existence and shapes of profiles are not presently available for many of these particularly interesting shock waves; one motivation for us to design the numerical approach presented here consisted in the wish to numerically obtain substantial new information on this problem.

\section{A parameterization \\ OF HIGHER-DIMENSIONAL HETEROCLINIC CONNECTIONS}

In the first part of this section, we consider a general system of ODE and introduce a parameterization for higher-dimensional heteroclinic connections and a 
notion of nondegeneracy of such manifolds. In the second part of the section we then focus on ODE systems that describe shock profiles for conservation laws and emphasize in particular the benefits of the new parameterization in that case.

2.1. The general case. Fix a vector field $F \in C^{2}\left(\mathbf{R}^{m}, \mathbf{R}^{m}\right)$ and consider a family $\Phi$ of trajectories which are heteroclinic to two given rest points $u^{-}, u^{+}$of $F$; in other words, $\phi \in \Phi$ satisfies

$$
\dot{\phi}=F(\phi), \quad \phi( \pm \infty)=u^{ \pm} .
$$

We assume that the intersection $M$ of $\mathcal{M}^{u}\left(u^{-}\right)$and $\mathcal{M}^{s}\left(u^{+}\right)$, given by

$$
M=\{\phi(x) \mid \phi \in \Phi, x \in(-\infty, \infty)\},
$$

is a smooth manifold of dimension $d$ for $d \in\{1, \ldots, m\}$.

For the purpose of its numerical approximation we parameterize $\Phi$ in the following specific way: Define a mapping

$$
\Omega: \Phi \rightarrow \mathbf{R}^{m}
$$

through

$$
\Omega(\phi) \equiv \int_{\mathbf{R}} A(x, \phi(x))\left(\phi(x)-\phi_{*}(x)\right) d x,
$$

with some appropriate function $A: \mathbf{R} \times \mathbf{R}^{m} \rightarrow \mathbf{R}^{m \times m}$ and $\phi_{*}$ either an element of $\Phi$ or given by

$$
\phi_{*}=\left\{\begin{array}{l}
u^{-}: x<0 \\
u^{+}: x>0
\end{array}\right.
$$

The subsequent assumption, crucial for the approach, means that $\Omega$ is a chart of $\Phi$.

Assumption 2.1. The mapping $\Omega$ is injective and the range $S=\Omega(\Phi)$ is a $d$ dimensional manifold in $\mathbf{R}^{m}$ allowing for a global chart $\mathbf{P}: S \rightarrow T \equiv \mathbf{P}(S) \subset \mathbf{R}^{d}$. The corresponding parameterization of $\Phi$ as $\left\{\phi^{\tau}\right\}_{\tau \in T}$ with $\phi^{\tau}$ defined through

$$
\mathbf{P} \Omega\left(\phi^{\tau}\right)=\tau, \tau \in T,
$$

is differentiable.

Remark 2.2. (i) Choosing different values of $\tau$ corresponds to switching between different trajectories $\phi^{\tau} \in \Phi$ but not necessarily between different orbits. One can think of $\tau$ as a pair $(\check{\tau}, \hat{\tau}) \in \mathbf{R} \times \mathbf{R}^{d-1}$ such that variations in $\check{\tau}$ at constant $\hat{\tau}$ result in a phase shift along one specific orbit while changing $\hat{\tau}$ corresponds to switching between orbitally different trajectories.

For $d=1$, shifts are the only possible variations. This case is treated by the work of Beyn[3]. He uses the condition

$$
\int_{-\infty}^{\infty} \dot{\phi}_{*}^{\top}\left(\phi-\phi_{*}\right) d x=0,
$$

which was also introduced by Doedel. In (2.4) a shift is selected so as to minimize the $L^{2}$-distance to some reference object $\phi_{*}$. Note the LHS in (2.4) can be recovered as a special case of the mapping $\mathbf{P} \circ \Omega$. However, we are interested in nondecaying $A$, in particular the case $A=I d$, which corresponds to $L^{1}$-difference rather than $L^{2}$-distance. 
(ii) In 2] $d$-dimensional heteroclinic manifolds that arise in the context of phase transition problems are considered. For parametrization, condition (2.4) together with $d-1$ conditions on the $L^{2}$-norm of the trajectories are used. While $L^{2}$ seems a natural setting in that case, for viscous conservation laws $L^{1}$ is the relevant space.

(iii) In practice it might be difficult to actually find a chart $\mathbf{P}$, since $S$ is not known in general.

Locally however any linear map $\mathbf{P}: \mathbf{R}^{m} \rightarrow \mathbf{R}^{d}$ of full rank with

$$
\mathcal{T} S \cap \mathrm{N}(\mathbf{P})=\{0\}
$$

yields such a chart by simple restriction to $S$.

By the parameterization induced by Assumption 2.1 we can view a single trajectory of $\Phi$ as the solution of a boundary value problem. This problem consists in finding, for $\tau \in \mathbf{R}^{d}$, a function $\phi^{\tau} \in C^{1}(\mathbf{R})$ such that

$$
\mathcal{F}^{\tau}\left(\phi^{\tau}\right)=\mathbf{0},
$$

where the operator $\mathcal{F}^{\tau}$ is defined by

$$
\mathcal{F}^{\tau}:\left\{\begin{aligned}
C^{1}(\mathbf{R}) & \rightarrow C^{0}(\mathbf{R}) \times \mathbf{R}^{d}, \\
\phi & \mapsto\left(\begin{array}{c}
\dot{\phi}-F(\phi) \\
\Psi(\phi)-\tau
\end{array}\right),
\end{aligned}\right.
$$

with $\Psi=\mathbf{P} \circ \Omega$.

Let us restrict our discussion to situations where the family $\Phi$ can be parameterized as introduced above and satisfies a certain genericity assumption.

Definition 2.3. Let Assumption 2.1 be true and let $\Phi$ be represented by the smooth $d$-parameter family

$$
\Phi=\left\{\phi^{\tau} \in C^{1}(\mathbf{R}) \mid \mathcal{F}^{\tau}\left(\phi^{\tau}\right)=\mathbf{0}, \tau \in T\right\} .
$$

Then $\Phi$ is said to constitute a nondegenerate manifold $M$ given by (2.1) if

(i) the rest points $u^{ \pm}$are hyperbolic; i.e., the Jacobian $D F$ of $F$ evaluated at $u^{ \pm}$ has no purely imaginary eigenvalues:

$$
\operatorname{spec}\left(D F\left(u^{ \pm}\right)\right) \subset \mathbf{C} \backslash i \mathbf{R},
$$

(ii) the relation $d=m_{u}^{-}+m_{s}^{+}-m$ holds, where $m_{u}^{-}=\operatorname{dim} \mathcal{M}^{u}\left(u^{-}\right)$and $m_{s}^{+}=$ $\operatorname{dim} \mathcal{M}^{s}\left(u^{+}\right)$

(iii) for each $\tau \in T$, the condition

$$
\dot{y}=D F\left(\phi^{\tau}\right) y, y \in C^{1}(\mathbf{R}), y( \pm \infty)=0 \Leftrightarrow y \in \operatorname{span}\left\{\frac{\partial \phi^{\tau}}{\partial \tau_{1}}, \ldots, \frac{\partial \phi^{\tau}}{\partial \tau_{d}}\right\}
$$

holds.

Definition 2.3$]$ is a generalization of a notion of nondegenerate orbits given by Beyn [3]. It will be substantial for verifying the error estimates that will be derived in Section 3 with a linearization technique. 
2.2. The conservation law case. In this section we focus on the treatment of viscous profiles; this means, for a shock wave solution (1.3) traveling with speed $s$, we consider the boundary value problem (1.8). For simplicity we think again of $\mathcal{D}=\delta I d, \delta>0$, while again the same analysis continues to hold for physically relevant systems with different, even degenerate viscosity matrix $\mathcal{D}$. We consider families $\Phi$ of viscous profiles and let $\Omega$ be defined as in (2.2) with $A=I d$. It is shown that this choice allows for a natural interpretation of Assumption 2.1.

Let $w^{ \pm}\left(., ., \kappa^{ \pm}\right)$denote the diffusion waves (14] $)$with base states $u^{ \pm}$and masses

$$
\int_{\mathbf{R}} w^{ \pm}\left(x, t, \kappa^{ \pm}\right) d x=\kappa^{ \pm} \in \mathcal{R}^{ \pm}\left(u^{ \pm}, s\right) .
$$

Here the eigenspace $\mathcal{R}^{ \pm}$is given by $\mathcal{R}^{ \pm}(u, s) \equiv \sum_{ \pm\left(\lambda_{i}(u)-s\right)>0} \mathrm{~N}\left(D f(u)-\lambda_{i}(u) I d\right)$.

$\phi_{*} \in \Phi$ is said to be asymptotically stable if for any function $\bar{u}$ in an interestingly large subclass of $L^{1}(\mathbf{R}) \cap L^{\infty}(\mathbf{R})$, there exist $\phi \in \Phi$ and $\kappa^{-}, \kappa^{+} \in R^{ \pm}\left(u^{ \pm}, s\right)$ such that the solution $u$ of (1.7) to the initial datum $\phi_{*}+\bar{u}$ exists and satisfies

$$
\lim _{t \rightarrow \infty}\left\|u(., t)-\left(\phi(.-s t)+w^{-}\left(., t, \kappa^{-}\right)+w^{+}\left(., t, \kappa^{+}\right)\right)\right\|_{L^{1}(\mathbf{R})}=0 ;
$$

by conservation of mass, (2.9) implies

$$
\begin{aligned}
\int_{\mathbf{R}} \bar{u}(x) d x & =\lim _{t \rightarrow \infty} \int_{\mathbf{R}} u(t, x)-\phi_{*}(x-s t) d x \\
& =\int_{\mathbf{R}} \phi(x)-\phi_{*}(x) d x+\kappa^{-}+\kappa^{+} .
\end{aligned}
$$

In many situations it is either known or generally believed (e.g., for physical reasons) that for a given shock wave profiles exist, are asymptotically stable and allow for a unique determination of the orbit $\phi$ by the mass of the initial perturbation. Then it is impossible to have $\Omega\left(\phi_{1}\right)=\Omega\left(\phi_{2}\right)$ for two different profiles $\phi_{1}, \phi_{2} \in \Phi$, as otherwise $\phi_{1,2}=\phi_{*}+\bar{u}_{1,2}$ with $\int \bar{u}_{1}=\int \bar{u}_{2}$ while the limits of the solutions $\phi_{1}, \phi_{2}$ do clearly not coincide. In other words, any such situation is an example of injectivity of $\Omega$. We recall two specific examples which admit a rigorous analysis.

Example 2.4 (Laxian shock wave). Let the eigenvalues $\lambda_{i}$ of $D f$ be simple and either genuinely nonlinear $\left(r_{i} \cdot \nabla \lambda_{i} \neq 0\right)$ or generically degenerate $\left(\left(r_{i} \cdot \nabla\right)^{2} \lambda \neq 0\right.$ whenever $\left.r_{i} \cdot \nabla \lambda_{i}=0\right)$. We consider the case of an $i$-shock $U$; that is, $k^{-}, k^{+}$in (1.5), (1.6) satisfy $i=m-k^{-}+1=k^{+}$. This means $d=1$ in the notation of Section 2.1 .

Then for $\left|u^{+}-u^{-}\right|$sufficiently small the viscous profile $\phi_{*}$ for $U$ exists and is asymptotically stable towards all perturbations in an appropriate subspace of $H^{1}(\mathbf{R})$. The asymptotic profile is given by $\phi=\phi_{*}\left(.+h_{i}\right)$ where $h=\left(h_{1}, \ldots, h_{m}\right)$ is the unique solution of

$$
\left[r_{1}\left(u^{-}\right)|\ldots| r_{i-1}\left(u^{-}\right)\left|u^{+}-u^{-}\right| r_{i+1}\left(u^{+}\right)|\ldots| r_{m}\left(u^{+}\right)\right] h^{\top}=\int_{\mathbf{R}} u_{0} .
$$

Note that the invertibility of the matrix in (2.11) is ensured for small jumps. For the proof of these results we refer to [14, [18] and [10].

Concerning overcompressive shock waves there is the following result.

Example 2.5 (Rotationally invariant system). Let us consider the system

$$
u_{t}+\left(|u|^{2} u\right)_{x}=\nu u_{x x}, u \in \mathbf{R}^{m}, \nu>0,
$$


of parabolic conservation laws. The eigenvalues of $D f(u)$ for $f(u)=|u|^{2} u$ are given by

$$
\lambda_{1}(u)=\cdots=\lambda_{m-1}(u)=|u|^{2}, \lambda_{m}(u)=3|u|^{2} .
$$

Consequently (2.12) is not strictly hyperbolic and allows for a variety of shock waves of different types. Here we restrict our discussion to waves $U$ with speed $s$ such that

$$
\lambda_{m}\left(u^{+}\right)<s<\lambda_{1}\left(u^{-}\right) .
$$

For this overcompressive situation we obtain $d=m$ by (1.5), (1.6) and (1.10). A $d$-parameter family of profiles for $U$ exists. The asymptotic stability of some of these profiles and the local bijectivity of the mapping $\int_{R} u_{0} \mapsto \phi$ were proved in [6]. Model (2.12) captures the typical behaviour of rotationally invariant systems at symmetry invariant points of their state spaces, e.g., for magnetohydrodynamics at the points with double eigenvalues. For the model character of (2.12), cf. [6].

Finally we mention the conjecture that the overcompressive shocks occurring in magnetohydrodynamics - our main example below - are all stable and satisfy Assumption 2.1 with $A=I d$; i.e., one can distinguish between different orbits which are heteroclinic to the same pair of rest points by their relative masses.

Examples of systems with families of overcompressive shock profiles which do not share these stability properties (including perturbed versions of the cubic model presented in Example 2.5) are given in [9]. It is for such cases that using $A \neq I d$ may be interesting. Analogous physical examples are not known to us at present.

\section{Approximation of Viscous profiles on finite intervals}

To develop a numerical method for the approximation of solutions of the problem (2.5) on the real line we will introduce an approximate boundary value problem on a finite interval. To obtain a well posed problem we introduce boundary conditions for the approximate problem.

3.1. Truncation to a finite interval. Let a finite interval $I=\left[X_{-}, X_{+}\right]$for $X_{-}<0<X_{+}$be given. If we consider the solution $\phi^{\tau}$ of the boundary value problem (2.5) we have, for $X_{-}, X_{+}$big enough,

$$
\phi^{\tau}\left(X_{-}\right) \in \mathcal{M}_{\text {loc }}^{u}\left(u^{-}\right), \quad \phi^{\tau}\left(X_{+}\right) \in \mathcal{M}_{\text {loc }}^{s}\left(u^{+}\right) .
$$

For a computational approach we have to approximate the local invariant manifolds $\mathcal{M}_{\text {loc }}^{u}\left(u^{-}\right)$and $\mathcal{M}_{\text {loc }}^{s}\left(u^{+}\right)$. To this end we substitute suitable asymptotic boundary conditions for the conditions (3.1) . Expressing these conditions by means of functions $b_{-} \in C^{2}\left(\mathbf{R}^{m}, \mathbf{R}^{m_{s}^{-}}\right), b_{+} \in C^{2}\left(\mathbf{R}^{m}, \mathbf{R}^{m_{u}^{+}}\right)$we require the approximant $\phi_{I}^{\tau} \in C^{1}\left(I, \mathbf{R}^{m}\right)$ of $\phi^{\tau}$ on $I$ to satisfy

$$
b_{-}\left(\phi_{I}^{\tau}\left(X_{-}\right)\right)=0, \quad b_{+}\left(\phi_{I}^{\tau}\left(X_{+}\right)\right)=0 .
$$

The special choice of the dimensions of the ranges of $b_{ \pm}$will become clear from a specific choice we make below, namely the so-called projection boundary conditions, where $b_{ \pm}$are linear.

We consider the characteristic decompositions

$$
D F\left(u^{ \pm}\right)=R\left(u^{ \pm}\right) \Lambda\left(u^{ \pm}\right) R\left(u^{ \pm}\right)^{-1}=L^{-1}\left(u^{ \pm}\right) \Lambda\left(u^{ \pm}\right) L\left(u^{ \pm}\right),
$$

where $\Lambda\left(u^{ \pm}\right)$is the Jordan canonical form of $D F\left(u^{ \pm}\right)$with Jordan blocks corresponding to eigenvalues of negative (positive) real part in the upper left (lower right) 
corner. $R\left(u^{ \pm}\right)$and $L\left(u^{ \pm}\right)$denote the matrices consisting of the right and left eigenvectors of $D F\left(u^{ \pm}\right)$. Each of these matrices is a juxtaposition of two submatrices which represent the stable and unstable subspaces of $D F\left(u^{ \pm}\right)$and $D F\left(u^{ \pm}\right)^{\top}$ :

$$
R\left(u^{ \pm}\right)=\left(R^{s}\left(u^{ \pm}\right) \mid R^{u}\left(u^{ \pm}\right)\right) \text {and } L\left(u^{ \pm}\right)=\left(\frac{L^{s}\left(u^{ \pm}\right)}{L^{u}\left(u^{ \pm}\right)}\right)
$$

For the projection boundary conditions, $\mathcal{M}_{l o c}^{u / s}\left(u^{ \pm}\right)$are approximated by the tangent spaces of $\mathcal{M}^{u / s}\left(u^{ \pm}\right)$in $u^{ \pm}$denoted by $\mathcal{T} \mathcal{M}^{u / s}\left(u^{ \pm}\right)$. Thus we require

$$
\phi_{I}^{\tau}\left(X_{-}\right) \in \mathcal{T} \mathcal{M}^{u}\left(u^{-}\right), \quad \phi_{I}^{\tau}\left(X_{+}\right) \in \mathcal{T} \mathcal{M}^{s}\left(u^{+}\right) .
$$

Since we have $\mathrm{R}\left(R^{s}\left(u^{+}\right)\right)=\mathrm{N}\left(\left(L^{u}\left(u^{+}\right)\right)\right)$, we can rewrite the condition $\phi_{I}^{\tau}\left(X_{+}\right) \in$ $\mathcal{T} \mathcal{M}^{s}\left(u^{+}\right)$in the form (3.2) by

$$
\left(L^{u}\left(u^{+}\right)\right)\left(\phi_{I}^{\tau}\left(X_{+}\right)-u^{+}\right)=0 \text {. }
$$

Note that $L^{u}\left(u^{+}\right) \in \mathbf{R}^{m \times m_{u}^{+}}$and (3.4) gives $m_{u}^{+}$conditions. The boundary condition in $X_{-}$can be treated analogously.

In the computations to be presented in Section 5 we exclusively use projection boundary conditions. We refer to [3] 4] for a discussion of the benefits due to projection boundary conditions. More general asymptotic boundary conditions of the form (3.2) were introduced in [4] 13, 15] in the framework of problems on semi-infinite intervals. The extension to infinite intervals was performed in [3] 5 .

The generalized phase condition $\Psi$ will be substituted for the approximate problem by $\Psi_{I}: C^{0}(I) \rightarrow \mathbf{R}^{d}$ with

$$
\Psi_{I}(\phi)=\mathbf{P} \int_{X_{-}}^{X_{+}} A(x, \phi(x))\left(\phi(x)-\phi_{*}(x)\right) d x
$$

To abbreviate the approximate problem, consider now the operator $\mathcal{F}_{I}^{\tau}$ with

$$
\mathcal{F}_{I}^{\tau}:\left\{\begin{aligned}
C^{1}(I) & \rightarrow C^{0}(I) \times \mathbf{R}^{m_{s}^{-}} \times \mathbf{R}^{m_{u}^{+}} \times \mathbf{R}^{d}, \\
\phi & \mapsto\left(\begin{array}{c}
\dot{\phi}-F(\phi) \\
b_{-}\left(\phi\left(X_{-}\right)\right) \\
b_{+}\left(\phi\left(X_{+}\right)\right) \\
\Psi_{I}(\phi)-\tau
\end{array}\right) .
\end{aligned}\right.
$$

Finally the approximate problem to (2.5) is to find, for $\tau \in \mathbf{R}^{d}$, a function $\phi_{I}^{\tau} \in$ $C^{1}(I)$ such that

$$
\mathcal{F}_{I}^{\tau}\left(\phi_{I}^{\tau}\right)=\mathbf{0}
$$


For later purpose we mention that the Fréchet derivative $\mathcal{F}_{I}^{\tau^{\prime}}(\phi)$ of $\mathcal{F}_{I}^{\tau}$ exists at every $\phi \in C^{1}(I)$ and is continuous for $A$ smooth enough:

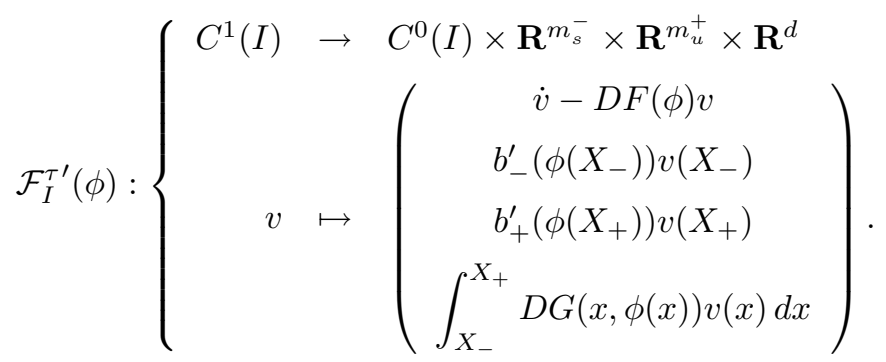

$D G(x,),. x \in \mathbf{R}$, denotes the Jacobian of the mapping $\xi \mapsto A(x, \xi)\left(\xi-\phi_{*}(x)\right)$, $\xi \in \mathbf{R}$.

\section{ERROR ANALYSIS}

4.1. The result. The error introduced by the truncation is analyzed. Due to the hyperbolicity of the rest points, the exponential convergence of the solutions of the restricted boundary value problem for $X_{ \pm} \rightarrow \pm \infty$ can be established. For the error analysis we consider the general case as introduced in subsection 2.1

Theorem 4.1. Let $A$ be a bounded $C^{1}$-mapping with bounded derivatives. Let Assumption 2.1 be true and $\Phi$ be represented by the smooth d-parameter family

$$
\Phi=\left\{\phi^{\tau} \in C^{1}(\mathbf{R}) \mid \mathcal{F}^{\tau}\left(\phi^{\tau}\right)=\mathbf{0}, \tau \in T\right\} .
$$

Assume further that for every $\tau \in T$ there exists an $\tilde{X}_{\tau}$ such that for any $I=$ $\left[X_{-}, X_{+}\right]$with $\left|X_{-}\right|, X_{+}>\tilde{X}_{\tau}$, the boundary conditions $b_{ \pm}$satisfy

$$
\begin{gathered}
b_{ \pm}\left(u^{ \pm}\right)=0, \\
\operatorname{det}\left(b_{-}^{\prime}\left(\phi^{\tau}\left(X_{-}\right)\right) R^{s}\left(u^{-}\right)\right) \neq 0, \quad \operatorname{det}\left(b_{+}^{\prime}\left(\phi^{\tau}\left(X_{+}\right)\right) R^{u}\left(u^{+}\right)\right) \neq 0 .
\end{gathered}
$$

Then for each $\tau \in T$ there exists an $X_{\tau}>\tilde{X}_{\tau}$ such that for any $I=\left[X_{-}, X_{+}\right]$with $\left|X_{-}\right|, X_{+}>\bar{X}_{\tau}$ the following is true:

(i) There is a $\delta>0$ such that a unique solution $\phi_{I}^{\tau} \in C^{1}(I)$ of (3.6) in $B_{\delta}\left(\phi^{\tau}\right)$ exists.

(ii) There is a constant $C>0$ such that:

$$
\left\|\phi_{I}^{\tau}-\phi^{\tau}\right\|_{C^{1}(I)} \leq C\left(X_{+}-X_{-}\right) \exp \left(-\min \left\{\lambda^{-} X_{-},-\lambda^{+} X_{+}\right\}\right),
$$

where $\lambda^{-}$and $\lambda^{+}$are given by the minimal absolute value of the real parts of the unstable (stable) eigenvalues of DF at $u^{-}\left(u^{+}\right)$.

The constant $C=C\left(\phi^{\tau}, \phi_{*}, A, \mathbf{P}, b_{ \pm}\right)$in (4.1) does not depend on $X_{ \pm}$.

The proof of Theorem 4.1 will be given in Section 4.3 below. Let us make some comments on this result. Other estimates on numerical schemes for heteroclinic or homoclinic connections as in [3, 5] typically lead to expressions for the error where the algebraic factor $X_{+}-X_{-}$does not appear. The reason for our slightly worse result is that we have to compensate for the fact that the operator $\Psi_{I}$ is not necessarily bounded uniformly with respect to $X_{ \pm}$; e.g., $\Psi_{I}$ is not bounded for the important choice for $A$ to be the unit matrix. However the algebraic factor $X_{+}-X_{-}$does not increase the asymptotic error significantly. 
Estimate (4.1) is not uniform in the parameter $\tau$. However this property cannot be expected: The constant $C$ typically increases for trajectories near the boundary of the heteroclinic manifold $M$. In Section 5 we present a numerical example for this fact.

4.2. Preliminaries for the error analysis. In this subsection we collect some results which will be used to establish the existence and convergence of solutions $\phi_{I}^{\tau}$ of (3.6) if the endpoints of the interval $I$ tend to $\pm \infty$. To be specific we recall some statements from the theory of exponential dichotomies for linear differential operators and a perturbation lemma to handle the convergence problem by a linearization technique.

Let the assumptions of Theorem 4.1 be valid and fix $\tau \in T$. Then consider the linear differential operator

$$
L: \mathcal{B}^{1} \rightarrow \mathcal{B}^{0}, \quad(L v)(x)=\dot{v}(x)-D F\left(u^{ \pm}\right) v(x),
$$

where $\mathcal{B}^{0}$ denotes the space of bounded functions $v \in C\left(\mathbf{R}, \mathbf{R}^{m}\right)$ and $\mathcal{B}^{1}$ the space of bounded functions $v \in C^{1}\left(\mathbf{R}, \mathbf{R}^{m}\right)$ with bounded derivatives of first order. Furthermore let $Y(x)$ be the associated fundamental matrix, identified by $Y(0)=I d$. By the hyperbolicity of the matrices $D F\left(u^{ \pm}\right)$and the smoothness of $D F\left(\phi^{\tau}().\right)$ we obtain

Lemma 4.2. The operator $L$ has an exponential dichotomy on the semi-infinite intervals $[0, \infty)$ and $(-\infty, 0]$; i.e., there exist projection matrices $P, Q \in \mathbf{R}^{m \times m}$ and positive constants $K, \alpha$ such that

$$
\begin{aligned}
\left|Y\left(x^{\prime}\right) P Y^{-1}(x)\right| \leq K \exp \left(-\alpha\left(x^{\prime}-x\right)\right) \quad\left(x^{\prime}, x \in[0, \infty), x^{\prime} \geq x\right), \\
\left|Y\left(x^{\prime}\right)(I-P) Y^{-1}(x)\right| \leq K \exp \left(-\alpha\left(x-x^{\prime}\right)\right) \quad\left(x^{\prime}, x \in[0, \infty), x \geq x^{\prime}\right), \\
\left|Y\left(x^{\prime}\right) Q Y^{-1}(x)\right| \leq K \exp \left(-\alpha\left(x^{\prime}-x\right)\right) \quad\left(x^{\prime}, x \in(-\infty, 0], x^{\prime} \geq x\right), \\
\left|Y\left(x^{\prime}\right)(I-Q) Y^{-1}(x)\right| \leq K \exp \left(-\alpha\left(x-x^{\prime}\right)\right) \quad\left(x^{\prime}, x \in(-\infty, 0], x \geq x^{\prime}\right) .
\end{aligned}
$$

We note that

$$
\begin{aligned}
& \mathrm{R}(P)=\left\{\eta \in \mathbf{R}^{m} \mid Y(.) \eta \text { is bounded in }[0, \infty)\right\}, \\
& \mathrm{N}(Q)=\left\{\eta \in \mathbf{R}^{m} \mid Y(.) \eta \text { is bounded in }(-\infty, 0]\right\} .
\end{aligned}
$$

Any projectors with the property (4.3) satisfy the inequalities (4.2). Furthermore $\mathrm{N}(L)$ can be represented by

$$
\mathrm{N}(L)=\{Y(.) \eta \mid \eta \in \mathrm{R}(P) \cap \mathrm{N}(Q)\} .
$$

For background material on exponential dichotomies and especially the proofs of the above statements, we refer to Palmer [17] and the literature cited therein.

The announced perturbation lemma can be found for example in Vainikko 20].

Lemma 4.3. Let $\left(Y,\|\cdot\|_{Y}\right),\left(Z,\|\cdot\|_{Z}\right)$ be Banach spaces, and $B_{\delta}\left(y_{0}\right)$ be given by $B_{\delta}\left(y_{0}\right)=\left\{y \in Y \mid\left\|y-y_{0}\right\|_{Y} \leq \delta\right\}$ for $\delta>0, y_{0} \in Y$.

Assume that the $C^{1}$-mapping $F: B_{\delta} \rightarrow Z$ satisfies for some constants $\sigma>\kappa>0$

(i) $F^{\prime}\left(y_{0}\right)$ is a homeomorphism,

$$
\text { (ii) } \quad\left\|F^{\prime}(y)-F^{\prime}\left(y_{0}\right)\right\| \leq \leqslant<\sigma \leq\left\|F^{\prime}\left(y_{0}\right)^{-1} \mid\right\|^{-1} \quad \forall y \in B_{\delta}\left(y_{0}\right) \text {, }
$$

(iii) $\left\|F\left(y_{0}\right)\right\|_{Z} \leq(\sigma-\kappa) \delta$. 
Then $F$ has a unique zero $\bar{y} \in B_{\delta}\left(y_{0}\right)$ and the estimate

$$
\left\|\bar{y}-y_{0}\right\|_{Y} \leq \frac{\left\|F\left(y_{0}\right)\right\|_{Z}}{(\sigma-\kappa)}
$$

holds. Here ||$|\cdot|||$ denotes the associated operator norm.

4.3. The proof of Theorem 4.1. In this section $C, C_{1}, C_{2}, C_{3}>0$ will denote generic constants which may depend on $\phi^{\tau}, \phi_{*}, A, \mathbf{P}, b_{ \pm}$but not on $X_{ \pm}$.

We will apply Lemma 4.3 to the mapping $\mathcal{F}_{I}^{\tau}$ given by (3.5). Following the notions in Lemma 4.3 we choose with natural norms

$$
Y=C^{1}(I), \quad Z=C^{0}(I) \times \mathbf{R}^{m_{s}^{-}+m_{u}^{+}+d}=C^{0}(I) \times \mathbf{R}^{m} .
$$

For $z=\left(\xi, r_{-}, r_{+}, \bar{r}\right) \in Z$ define $\|\cdot\|_{Z}$ by $\|\cdot\|_{Z}=\|\xi\|_{0}+\left|r_{+}\right|+\left|r_{-}\right|+|\bar{r}|$. For $y_{0}$ we take the exact solution $\phi^{\tau}$ of the problem (2.5) restricted to the interval $I$. To prove Theorem 4.1 it then remains to check conditions $(i), \ldots,($ iii $)$.

With the Fréchet derivative $\mathcal{F}_{I}^{\tau^{\prime}}\left(\phi^{\tau}\right)$ from (3.7) we consider the variational problem

$$
\mathcal{F}_{I}^{\tau^{\prime}}\left(\phi^{\tau}\right)(v)=\left(\xi, r_{-}, r_{+}, \bar{r}\right), \quad\left(\xi, r_{-}, r_{+}, \bar{r}\right) \in Z .
$$

From Lemma 4.4 below we deduce that a solution $u$ of the variational problem satisfies the stability estimate

$$
\|v\|_{1} \leq C_{1}\left(X_{+}-X_{-}\right)\left(\|\xi\|_{0}+\left|r_{+}\right|+\left|r_{-}\right|+|\bar{r}|\right)
$$

The linearity of $\mathcal{F}_{I}^{\tau^{\prime}}(\phi)$ ensures now that its inverse exists and is continuous at least on $\mathrm{R}\left(\mathcal{F}_{I}^{\tau^{\prime}}(\phi)\right)$. With $\sigma=\left(C_{1}\left(X_{+}-X_{-}\right)\right)^{-1}$ we get the bound

$$
\left\|\mathcal{F}_{I}^{\tau^{\prime}}(\phi)^{-1}\right\| \mid \leq \frac{1}{\sigma}
$$

Now let $\kappa=\sigma / 2$ and $\delta=\left(2 C_{1} C_{2}\left(X_{+}-X_{-}\right)\right)^{-1}$. Taking into account $F \in C^{2}$ we obtain for all $\tilde{\phi} \in B_{\delta}\left(\phi^{\tau}\right)$

$$
\|\| \mathcal{F}_{I}^{\tau^{\prime}}(\tilde{\phi})-\mathcal{F}_{I}^{\tau^{\prime}}\left(\phi^{\tau}\right)\left\|\leq C_{2}\right\| \tilde{\phi}-\phi^{\tau} \|_{1} \leq C_{2} \delta=\kappa<\sigma \leq \frac{1}{\left\|\mathcal{F}_{I}^{\tau^{\prime}}\left(\phi^{\tau}\right)^{-1}\right\|} .
$$

Thus conditions $(i)$ and $(i i)$ of Lemma 4.3 are satisfied.

Due to the hyperbolicity of the rest points $u^{-}$and $u^{+}$we have $\phi^{\tau}(x)-u^{-}=$ $\mathcal{O}\left(\exp \left(\lambda^{-} x\right)\right)$ for $x \rightarrow-\infty$ and $\phi^{\tau}(x)-u^{+}=\mathcal{O}\left(\exp \left(-\lambda^{+} x\right)\right)$ for $x \rightarrow \infty$. Since $A$ is bounded, the consistency estimate

$$
\begin{aligned}
\left\|\mathcal{F}_{I}^{\tau}\left(\phi^{\tau}\right)\right\|_{Z} \leq & \left|b_{-}\left(\phi^{\tau}\left(X_{-}\right)\right)\right|+\left|b_{+}\left(\phi^{\tau}\left(X_{+}\right)\right)\right| \\
& +\left|\int_{-\infty}^{X_{-}} A\left(x, \phi^{\tau}(x)\right)\left(\phi^{\tau}(x)-\phi_{*}(x)\right) d x\right| \\
& +\left|\int_{X_{+}}^{\infty} A\left(x, \phi^{\tau}(x)\right)\left(\phi^{\tau}(x)-\phi_{*}(x)\right) d x\right| \\
\leq & C_{3} \exp \left(-\min \left\{\lambda^{-} X_{-},-\lambda^{+} X_{+}\right\}\right)
\end{aligned}
$$

holds for $X_{-}, X_{+}$big enough. Recall here that the smoothness of the functions $b_{ \pm}$ and the property $b_{ \pm}\left(u^{ \pm}\right)=0$ implies $b_{ \pm}\left(\phi^{\tau}\left(X_{ \pm}\right)\right)=\mathcal{O}\left(\exp \left(\mp \lambda^{ \pm} X_{ \pm}\right)\right)$. 
Using this exponential decay property we can satisfy condition (ii) of Lemma 4.3 for a sufficiently large interval $I$; i.e., there exists a constant $X_{\tau}>0$ such that for $X_{-}, X_{+}>X_{\tau}$ we have

$$
\left\|\mathcal{F}_{I}^{\tau}\left(\phi^{\tau}\right)\right\|_{Z} \leq \frac{1}{4 C_{1}^{2} C_{2}} \frac{1}{\bar{X}^{2}} \leq(\sigma-\kappa) \delta .
$$

Lemma 4.4. Let the assumptions of Theorem 4.1 be valid and let $\left(\xi, r_{-}, r_{+}, \bar{r}\right) \in$ $C^{0}(I) \times \mathbf{R}^{m_{s}^{-} \times m_{u}^{+} \times d}$. As soon as $\left|X_{-}\right|, X_{+}$are sufficiently large, any solution $v \in$ $C^{1}(I)$ of the variational problem

$$
\mathcal{F}_{I}^{\tau^{\prime}}(\phi)(v)=\left(\xi, r_{-}, r_{+}, \bar{r}\right)
$$

satisfies

$$
\|v\|_{1} \leq C_{1}\left(X_{+}-X_{-}\right)\left(\|\xi\|_{0}+\left|r_{+}\right|+\left|r_{-}\right|+|\bar{r}|\right) .
$$

Proof. By Lemma 4.2 the linear operator $L$ has exponential dichotomies on $(-\infty, 0]$ and $[0, \infty)$. Consequently there are projectors $P^{\prime}$ and $Q^{\prime}$ such that the inequalities (4.2) hold. By the nondegeneracy property (2.8) and

$$
\mathrm{N}(L)=\left\{Y(.) \eta \mid \eta \in \mathrm{R}\left(P^{\prime}\right) \cap \mathrm{N}\left(Q^{\prime}\right)\right\}
$$

we have

$$
\mathrm{R}\left(P^{\prime}\right) \cap \mathrm{N}\left(Q^{\prime}\right)=\operatorname{span}\left\{\frac{\partial \phi^{\tau}}{\partial \tau_{1}}(0), \ldots, \frac{\partial \phi^{\tau}}{\partial \tau_{d}}(0)\right\} .
$$

This space will henceforth be denoted by $\mathcal{Z}_{1}$. Let the linear subspaces $\mathcal{Z}_{2}, \mathcal{Z}_{3} \subset \mathbf{R}^{m}$ be defined according to

$$
\mathrm{R}\left(P^{\prime}\right)=\mathcal{Z}_{1} \oplus \mathcal{Z}_{2}, \quad \mathrm{~N}\left(Q^{\prime}\right)=\mathcal{Z}_{1} \oplus \mathcal{Z}_{3} .
$$

Finally define the subspace $\mathcal{Z}_{4}$ via $\left(\mathrm{R}\left(P^{\prime}\right)+\mathrm{N}\left(Q^{\prime}\right)\right) \oplus \mathcal{Z}_{4}=\mathbf{R}^{m}$. Now let $P$ be the projector onto $\mathcal{Z}_{1} \oplus \mathcal{Z}_{2}, Q$ the projector onto $\mathcal{Z}_{2} \oplus \mathcal{Z}_{4}$, and $\Pi$ the projector onto $\mathcal{Z}_{1}$. Note that $P$ and $Q$ satisfy

$$
\mathrm{R}(P)=\mathrm{R}\left(P^{\prime}\right) \text { and } \mathrm{N}(Q)=\mathrm{N}\left(Q^{\prime}\right) .
$$

Consequently estimates (4.2) hold with $P$ on $[0, \infty)$ and $Q$ on $(-\infty, 0]$. by

Using $P$ and $Q$ consider now $s_{+}:[0, \infty) \rightarrow \mathbf{R}^{m}$ and $s_{-}:(-\infty, 0] \rightarrow \mathbf{R}^{m}$ given

$$
\begin{aligned}
& s_{+}(x)=Y(x) P \int_{0}^{x} Y\left(x^{\prime}\right)^{-1} \xi\left(x^{\prime}\right) d x^{\prime}-Y(x)(I-P) \int_{x}^{X_{+}} Y\left(x^{\prime}\right)^{-1} \xi\left(x^{\prime}\right) d x^{\prime}, \\
& s_{-}(x)=-Y\left(x^{\prime}\right)(I-Q) \int_{x}^{0} Y\left(x^{\prime}\right)^{-1} \xi\left(x^{\prime}\right) d x^{\prime}+Y(x) Q \int_{X_{-}}^{x} Y\left(x^{\prime}\right)^{-1} \xi\left(x^{\prime}\right) d x^{\prime} .
\end{aligned}
$$

Then the function

$$
s(x)=\left\{\begin{array}{lll}
s_{-}(x) & : & X_{-} \leq x<0 \\
s_{+}(x) & : & 0<x \leq X_{+}
\end{array}\right.
$$

is a particular solution of the linear ODE $L v=\xi$ in the interval $I \backslash\{0\}$ that satisfies $\|s\|_{0} \leq C\|\xi\|_{0}$ due to the exponential dichotomy of $L$ (Lemma 4.2). For $\eta_{-}, \eta_{+} \in \mathbf{R}^{m}$ we can thus represent the solution $u$ as follows.

$$
v(x)=Y(x) H_{\eta_{-}, \eta_{+}}(x)+s(x), \quad x \in I .
$$


Here $H_{\eta_{-}, \eta_{+}}: \mathbf{R} \rightarrow \mathbf{R}^{m}$ denotes the piecewise constant function that is equal to $\eta_{-}$in $\left(X_{-}, 0\right]$ and equal to $\eta_{+}$in $\left(0, X_{+}\right)$.

To finish the proof we have to estimate $\eta_{-}$and $\eta_{+}$. Therefore let

$$
N(x)=\left[\frac{\partial \phi^{\tau}}{\partial \tau_{1}}(x)|\ldots| \frac{\partial \phi^{\tau}}{\partial \tau_{d}}(x)\right] .
$$

The property (2.8) assures that there exists a vector $\tilde{\eta}_{ \pm} \in \mathbf{R}^{d}$ such that $\Pi \eta_{ \pm}=$ $N(0) \tilde{\eta}_{ \pm}$. Unique solvability of the linear initial value problem $L v=0, v(0)=\eta_{ \pm}$ guarantees for all $x \in \mathbf{R}$

$$
Y(x) \Pi \eta_{ \pm}=N(x) \tilde{\eta}_{ \pm}
$$

We rewrite (4.6) as

$$
v(x)-N(x) \tilde{\eta}_{ \pm}=Y(x)\left(\eta_{ \pm}-\Pi \eta_{ \pm}\right)+s_{ \pm}(x) .
$$

Let $e_{I}=\max \left\{\exp \left(\alpha X_{-}\right), \exp \left(-\alpha X_{+}\right)\right\}$with $\alpha$ from Lemma 4.2, Then if the estimates

$$
\begin{array}{ll}
\left|v(x)-N(x) \tilde{\eta}_{+}\right| \leq C\left(\|\xi\|_{0}+\left|r_{+}\right|+\left|r_{-}\right|+e_{I}\left|\tilde{\eta}_{+}\right|\right), & x \in\left[0, X_{+}\right), \\
\left|v(x)-N(x) \tilde{\eta}_{-}\right| \leq C\left(\|\xi\|_{0}+\left|r_{+}\right|+\left|r_{-}\right|+e_{I}\left|\tilde{\eta}_{-}\right|\right), & x \in\left(X_{-}, 0\right]
\end{array}
$$

hold, it remains to bound $\eta_{ \pm}$, respectively $\tilde{\eta}_{ \pm}$, in terms of $\|\xi\|_{0},\left|r_{ \pm}\right|$and $|\bar{r}|$ in order to verify (4.5). We postpone the verification of (4.8) to the end of the proof and collect some observations to achieve the bound on $\tilde{\eta}_{ \pm}$.

Obviously we have the representation

$$
\begin{aligned}
\int_{X_{-}}^{X_{+}} D G\left(x, \phi^{\tau}(x)\right) N(x) \tilde{\eta}_{+} d x= & \int_{X_{-}}^{0} D G\left(x, \phi^{\tau}(x)\right) N(x)\left(\tilde{\eta}_{+}-\tilde{\eta}_{-}\right) d x \\
& +\int_{X_{-}}^{X_{+}} D G\left(x, \phi^{\tau}(x)\right) N(x) H_{\tilde{\eta}_{+}, \tilde{\eta}_{-}} d x .
\end{aligned}
$$

The continuity of $u$ on the whole interval $I$ implies

$$
\left|\tilde{\eta}_{+}-\tilde{\eta}_{-}\right| \leq C\|s\|_{0}
$$

Returning to the estimation of $\tilde{\eta}_{+}$, Assumption 2.1 shows that, for $X_{+},\left|X_{-}\right|$sufficiently large, the $(d \times d)$-matrix

$$
\left[\mathbf{P} \int_{I} D G\left(x, \phi^{\tau}(x)\right) \frac{\partial \phi^{\tau}}{\partial \tau_{1}}|\ldots| \mathbf{P} \int_{I} D G\left(x, \phi^{\tau}(x)\right) \frac{\partial \phi^{\tau}}{\partial \tau_{d}}\right]
$$


is invertible. Combining this with (4.9), we obtain

$$
\begin{aligned}
\left|\tilde{\eta}_{+}\right| \leq & C\left|\mathbf{P} \int_{X_{-}}^{X_{+}} D G\left(x, \phi^{\tau}(x)\right) N(x) \tilde{\eta}_{+} d x\right| \\
\leq & C\left(\left|\int_{X_{-}}^{0} D G\left(x, \phi^{\tau}(x)\right) N(x)\left(\tilde{\eta}_{+}-\tilde{\eta}_{-}\right) d x\right|\right. \\
& \left.+\left|\int_{X_{-}}^{X_{+}} D G\left(x, \phi^{\tau}(x)\right)\left(N(x) H_{\tilde{\eta}_{+}, \tilde{\eta}_{-}}-v(x)\right) d x\right|\right) \\
& +\left|\mathbf{P} \int_{X_{-}}^{X_{+}} D G\left(x, \phi^{\tau}(x)\right) v(x) d x\right| \\
\leq & C\left(X_{+}-X_{-}\right)\left(\|\xi\|_{0}+\left|r_{+}\right|+\left|r_{-}\right|+|\bar{r}|+e_{I}\left|\tilde{\eta}_{+}\right|+e_{I}\left|\tilde{\eta}_{-}\right|\right) .
\end{aligned}
$$

The last estimate follows from (4.8) and (4.10) for $I$ sufficiently large. Combination with the analogous inequality for $\tilde{\eta}_{-}$gives the desired estimate for $\left|\tilde{\eta}_{+}\right|,\left|\tilde{\eta}_{-}\right|$for an eventually even larger interval $I$.

It remains to show the estimate (4.8). Therefore we note

$$
\begin{aligned}
\left|Y(x)(I-P) \eta_{+}\right| \leq C\left(\|\xi\|_{0}+\left|r_{+}\right|+e_{I}\left|\eta_{+}\right|\right), & x \in\left[0, X_{+}\right], \\
\left|Y(x) Q \eta_{-}\right| \leq C\left(\|\xi\|_{0}+\left|r_{-}\right|+e_{I}\left|\eta_{-}\right|\right), & x \in\left[X_{-}, 0\right] .
\end{aligned}
$$

The proof of these inequalities using the assumptions on $b_{ \pm}$can be found in [3], Appendix D.

Furthermore using the construction of the projectors $P, Q, \Pi$ we obtain the estimates

$$
|P \eta-\Pi \eta| \leq C|Q \eta|,
$$

for all $\eta \in \mathbf{R}^{m}$. Consider now for $x \in\left[0, X_{+}\right]$

$$
\left|Y(x)\left(\eta_{+}-\Pi \eta_{+}\right)\right| \leq\left|Y(x)(I-P) \eta_{+}\right|+\left|Y(x)\left(P \eta_{+}-\Pi \eta_{+}\right)\right| .
$$

Since $\left(P \eta_{+}-\Pi \eta_{+}\right) \in\left\{\eta \in \mathbf{R}^{m} \mid Y(x) \eta\right.$ bounded for $\left.x \geq 0\right\}$, we have from (4.12)

$$
\begin{aligned}
\left|Y(x)\left(\eta_{+}-\Pi \eta_{+}\right)\right| & \leq\left|Y(x)(I-P) \eta_{+}\right|+C\left|Q\left(\eta_{+}-\eta_{-}\right)\right|+C\left|Q \eta_{-}\right| \\
& \leq C\left(\left|r_{+}\right|+\left|r_{-}\right|+\|\xi\|_{0}+e_{I}\left|\eta_{+}\right|\right) .
\end{aligned}
$$

The last estimate follows from (4.11) and (4.10). In view of equation (4.7) the first equation in (4.8) is proven since by using the exponential dichotomies on $(-\infty, 0]$ and $[0, \infty)$ we can bound $\|s\|_{0}$ in terms of $\|\xi\|_{0}$.

\section{NumericAl EXPERIMENTS}

Before we present a number of numerical examples from the field of conservation laws, let us comment on some implementation details. The problem 3.6 can be readily rewritten as a two-point boundary value problem of dimension $m+d$ if 
we substitute the integral constraint $\Psi_{I}\left(\phi_{I}^{\tau}\right)-\tau=0$ in (3.6) by the differential equation

$$
\dot{w}=\mathbf{P} A\left(., \phi_{I}^{\tau}\right)\left(\phi_{I}^{\tau}-\phi_{*}\right), w(x) \in \mathbf{R}^{d},
$$

which has to be augmented by the boundary conditions

$$
w\left(X_{-}\right)=0 \text { and } w\left(X_{+}\right)=\tau .
$$

In all numerical computations presented below $\phi_{*}$ was chosen to be the jump function (2.3). Since we exclusively present results for viscous profiles, we set $A=I d$. Concerning the asymptotic boundary conditions note that the basis vectors of the stable and unstable manifolds were always known explicitly or easily computed. For the solution of the two-point boundary value problem we partly used the code COLNEW of Bader and Ascher [1. In nearly all cases the jump (2.3) turns out to be a good initial guess to start this collocation method.

5.1. A test example. We start the presentation of numerical results with a somewhat constructed problem, which simply leads to overcompressive shock waves and can be solved analytically. The main task is to illustrate the rates obtained by Theorem 4.1 .

For $i=1, \ldots, 3$ consider the (decoupled) system

$$
u_{t}^{i}+f\left(u^{i}\right)_{x}=\epsilon^{i} u_{x x}^{i}, \quad f\left(u^{i}\right)=\frac{1}{2}\left(u^{i}\right)^{2} .
$$

The components of the viscous profile $\phi=\left(\phi^{1}, \phi^{2}, \phi^{3}\right)$ to the overcompressive shock wave $U$ with

$$
u^{-}=(1,1,1), u^{+}=(-1,-1,-1), s=0,
$$

are solutions of the boundary value problem

$$
\varepsilon^{i} \phi^{i^{\prime}}=f\left(\phi^{i}\right)-\frac{1}{2}, \quad \phi^{i}( \pm \infty)=\mp 1 .
$$

Here the rest points $u^{ \pm}$are connected by a three-dimensional heteroclinic manifold which fills the unit cube. The associated trajectories are given for $\tau^{1}, \tau^{2}, \tau^{3} \in \mathbf{R}$ by

$$
\phi^{i}(x)=\tanh \left(\frac{1}{2 \epsilon^{i}}\left(x-\tau^{i}\right)\right), \quad i=1, \ldots, 3 .
$$

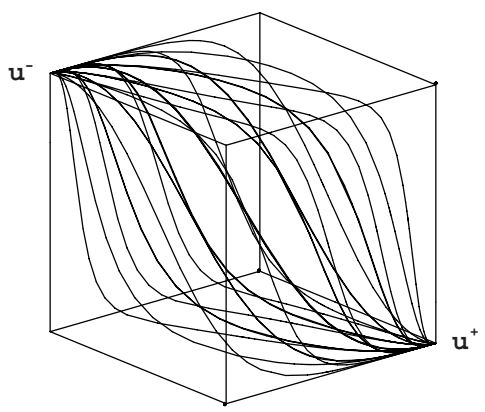

FiguRe 1. Some orbits from the three-dimensional manifold connecting $u^{-}$and $u^{+}$. 

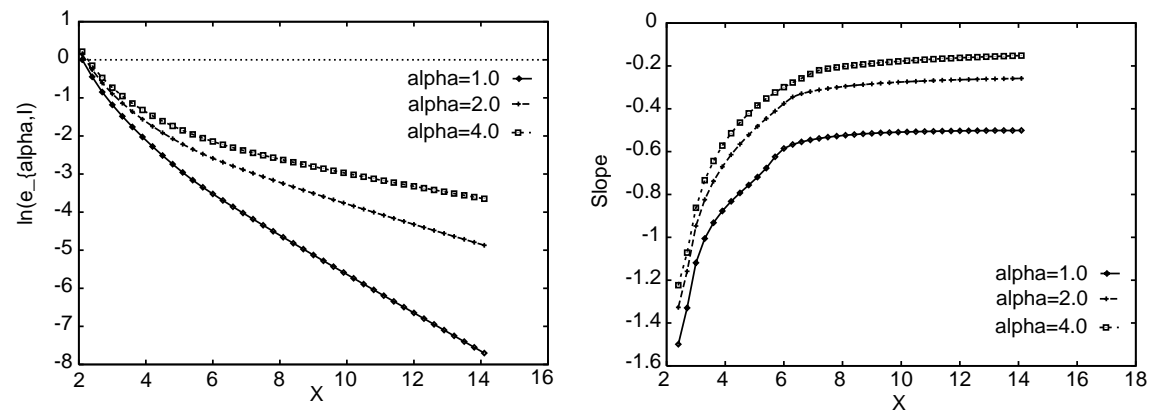

FiguRE 2. Logarithm of error and slope of logarithm of error versus length of $I$ for different scalings.

For illustration we present in Figure 1 some approximate orbits obtained on the interval $I=[-12,12]$. At first we demonstrate the exponential decay of the error as predicted by Theorem 4.1. For $\tau^{1}=1.0, \tau^{2}=0, \tau^{3}=2.0$ we fix some orbit $\phi_{\alpha}$ via the choice of different sets of viscosity parameters $\varepsilon^{1}(\alpha), \varepsilon^{2}(\alpha), \varepsilon^{3}(\alpha)$. For $\alpha=1.0,2.0,3.0$ let

$$
\epsilon^{1}=2.0 \alpha, \quad \epsilon^{2}=\alpha / 2.0, \quad \epsilon^{1}=1.0
$$

The left-hand picture in Figure 2 displays the logarithm of the error

$$
e_{\alpha, I}=\sum_{i=1}^{3}\left\|\phi_{I, \alpha}^{i}-\phi_{\alpha}^{i}\right\|_{L^{\infty}(\mathbf{R})}
$$

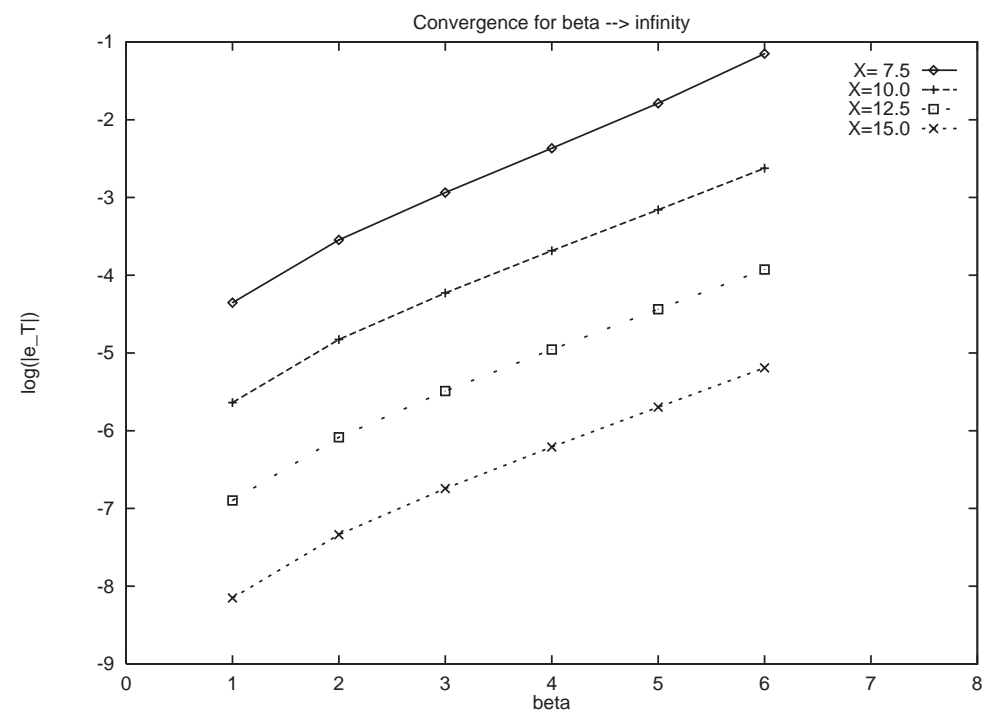

FigURE 3. Logarithm of the error $\left\|\phi_{I, \beta}-\phi_{\beta}\right\|_{L^{\infty}(\mathbf{R})}$ for different values of $\beta$ and $X$. 
in dependence of the bounded interval $I=[-X, X], X>0 . \phi_{I, \alpha}$ is extended to $\mathbf{R}$ by constant continuation with the values $\phi_{I, \alpha}( \pm X)$. The right-hand picture shows the slope of the same quantity. These numerical results confirm the estimate (4.1), at least the exponential decay rate $-\min \left\{\lambda^{-} X_{-},-\lambda^{+} X_{+}\right\}$for long time asymptotics. We obtain the expected rates $-1 / 2,-1 / 4,-1 / 6$, respectively.

Subsequently we illustrate that the estimate (4.1) in general cannot hold uniformly for all orbits of the heteroclinic manifold. Note that all vertices of the cube in Figure 1 are rest points for the dynamical system in (5.2). We consider a sequence of parameters $\tau_{\beta}^{1}, \tau_{\beta}^{2}, \tau_{\beta}^{3}$ such that the corresponding orbits $\phi_{\beta}$ approach some of these rest points for $\beta \rightarrow \infty$. As the bulk of the variation of the $\phi_{\beta}$ spreads on intervals of length growing proportionally to $\beta$, larger and larger $I$ 's are needed to well approximate $\phi_{\beta}$.

The settings are

$$
\begin{gathered}
\alpha=1.0, \tau_{\beta}^{1}=\beta, \tau_{\beta}^{2}=0.0, \tau_{\beta}^{3}=-\beta, \\
\beta=1.0,2.0,4.0,5.0,6.0 .
\end{gathered}
$$

Figure 3 shows the increase of the error for increasing values of $\beta$. Results for different intervals $I$ are displayed.

5.2. The rotationally invariant system. We consider the system (2.12) from Example 2.5 and obtain for $m=2$ the ODE-system

$$
\dot{u}=\left(|u|^{2}-s\right) u-q, u=\left(u^{1}, u^{2}\right) \in \mathbf{R}^{2} .
$$

Note that the uniform viscosity parameter $\nu$ is scaled out. For the choice $q \equiv$ $(0.05,0)$ and $s \equiv 0.95$ system (5.3) has three rest points: the source $u^{-}=(1,0)$, the sink $u^{1} \approx(-0.0528,0)$, the saddle $u_{2} \approx(-0.9472,0)$. There exists a pair of orbits from $u^{-}$to $u^{2}$ which is the boundary of a two-dimensional heteroclinic manifold connecting $u^{-}$and $u_{1}$. The shock wave $U$ with $u^{+}=u^{1}$ belongs to the class of overcompressive shocks discussed in Example 2.5.

Our algorithm is now used to approximate orbits from the manifold. The results can be found in Figure 4 In order to solve the associated boundary value problem with $\left|\phi_{I}^{\tau}\left(X_{ \pm}\right)-u^{ \pm}\right|<0.01$ we have been forced to choose $\left|X_{ \pm}\right|$up to 200 for the orbits most close to $u_{2}$ while $I=[-10,10]$ was sufficient for the orbit following the

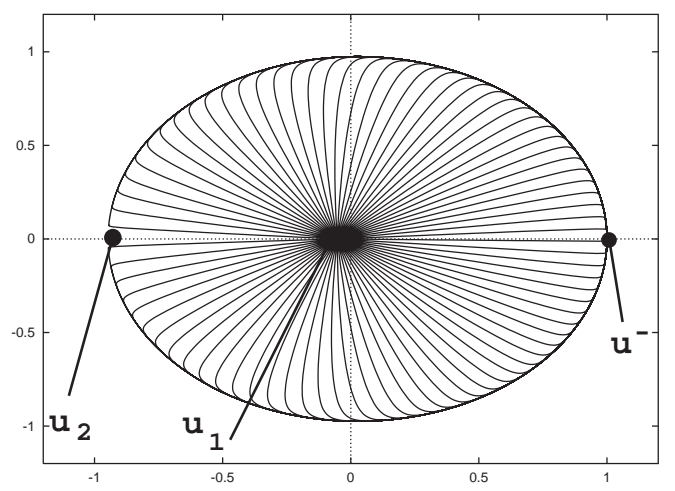

Figure 4. Two-dimensional heteroclinic manifold in the $u^{1} u^{2}$-plane. 
$u^{1}$-axis. These large intervals arise due to the slow dynamics of (5.3) near the pair of orbits connecting $u^{-}$and $u^{2}$.

5.3. Compressible Navier-Stokes equations. As a first physically relevant model we consider plane waves that are solutions of the compressible $1 D$-Euler equations which describe the dynamics of an inviscid fluid. We assume that the fluid is an ideal polytropic gas. Adding the natural dissipation mechanism we obtain the compressible Navier-Stokes equations.

The corresponding ODE-problem can be written after reformulation and rescaling as the $2 \times 2$-system

$$
\begin{aligned}
\lambda \dot{\tau} & =F(\theta, \tau) \equiv \tau+\frac{R \theta}{\tau}-j, \\
\kappa \dot{\theta} & =G(\theta, \tau) \equiv c_{V} \theta-\frac{\tau^{2}}{2}+j \tau-e .
\end{aligned}
$$

Here the unknowns $\tau$ and $\theta$ correspond to longitudinal velocity and temperature while $R>0$ is the gas constant, $c_{V}>0$ denotes specific heat at constant volume. $j, e$ are components of the vector $q$ (cf. (1.10) ). Note that due to Galilean invariance $s=0$ is assumed without loss of generality.

The only possible shock wave solutions of the Euler equations are of Laxian type. It is well known that all these shocks have viscous profiles for all $\lambda, \kappa>0$ [1]. Figure 5 shows the numerically obtained profiles for different ratios of $\lambda$ and $\kappa$ :

$$
\text { (a): } \frac{\lambda}{\kappa}=1,(\mathrm{~b}): \frac{\lambda}{\kappa}=1 / 100,(\mathrm{c}): \frac{\lambda}{\kappa}=10 .
$$

The other parameters are fixed with

$$
R=0.7, c_{V}=1.5, j=2.0, e=3.0 .
$$

This choice corresponds to a 3 -shock (cf. Example 2.4), where $u^{-}$is a source and $u^{+}$is a saddle.

The dotted lines in Figure 5 refer to the nullclines of $F$ (concave curve) and $G$ (convex curve). The computations were performed on $I=[-80,80]$. While the orbits will approach for $\lambda / \kappa \rightarrow \infty$ the nullcline of $G$, the orbits will not reach the

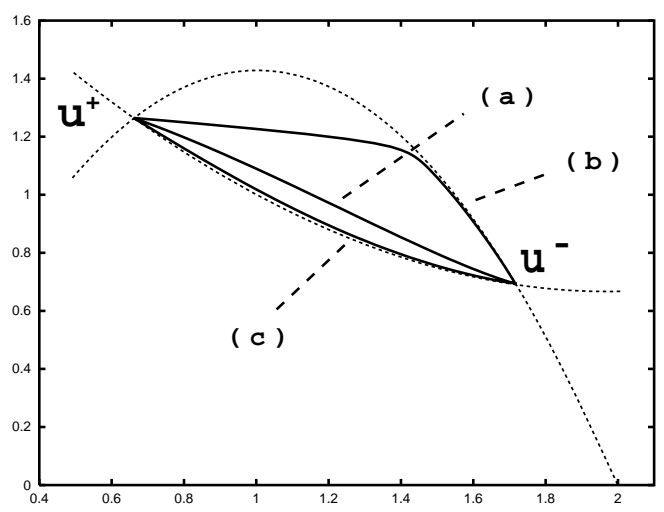

Figure 5. Viscous profiles for an Euler shock in the $\tau \theta$-plane (different ratios of viscosity parameters in accordance with (5.4)). 
nullcline of $F$ for $\lambda / \kappa \rightarrow 0$ indicating the existence of a subshock in this case. This is due to the fact that the derivative of $F$ with respect to $\tau$ will change sign on the curve segment between $u^{-}$and $u^{+}$.

5.4. The equations of viscous magnetohydrodynamics (MHD). While the Euler equations do not allow for overcompressive shock waves, these waves can be observed for the equations of magnetohydrodynamics, which describe the motion of electrically conducting fluids (for further discussion on these so-called intermediate waves we refer to [12, 21, 8]). If we consider the associated dissipative model including the effects of fluid viscosity, thermal conductivity and resistivity, the subsequent ODE-system can be obtained after reformulation and rescaling:

$$
\begin{aligned}
\nu \dot{\mathbf{b}} & =-d \mathbf{w}+\tau \mathbf{b}-\mathbf{c} \\
\lambda \dot{\tau} & =\tau+\frac{R \Theta}{\tau}+\frac{1}{2}|\mathbf{b}|^{2}-j, \\
\mu \dot{\mathbf{w}} & =\mathbf{w}-d \mathbf{b}, \\
\kappa \dot{\Theta} & =c_{v} \Theta-\frac{1}{2}\left(|\mathbf{w}|^{2}-2 d \mathbf{w} \cdot \mathbf{b}+\tau|\mathbf{b}|^{2}\right)-\frac{\tau^{2}}{2}+j \tau+\mathbf{b} \cdot \mathbf{c}-e .
\end{aligned}
$$

In this system of six equations, which we will refer to as $\Sigma^{6}$, the quantity $\bar{q}=$ $(d, \mathbf{c}, j, e) \in \mathbf{R} \times \mathbf{R}^{2} \times \mathbf{R} \times \mathbf{R}$ plays the rôle of $q$ in (1.8). The components of $(\mathbf{b}, \tau, \mathbf{w}, \Theta) \in \mathbf{R}^{2} \times(0, \infty) \times \mathbf{R}^{2} \times(0, \infty)$ stand for transverse magnetic field, longitudinal velocity, transverse velocity, and temperature. In addition to the dissipation coefficients $\lambda$ and $\kappa$ we already had in the electrically neutral case there are now also the dissipation parameters $\nu$ for resistivity and $\mu$ for "transversal" fluid viscosity. From the six-dimensional system $\Sigma^{6}$ we will restrict attention to the system $\Sigma^{3}$ that consists of the three equations

$$
\begin{aligned}
\nu \dot{\mathbf{b}} & =\left(\tau-d^{2}\right) \mathbf{b}-\mathbf{c}, \\
\lambda \dot{\tau} & =\frac{1}{2}|\mathbf{b}|^{2}+\tau-j+\frac{1}{\left(1+c_{V} / R\right) \tau}\left(-\frac{\tau^{2}}{2}-\frac{d^{2}}{2}|\mathbf{b}|^{2}-\mathbf{b} \cdot \mathbf{c}+e\right) .
\end{aligned}
$$

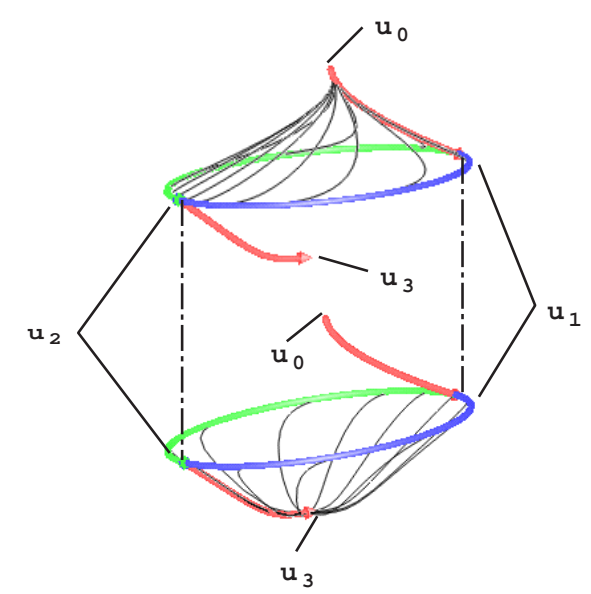

FiguRE 6. Upper and lower parts of the heteroclinic structure of $\Sigma^{3}$ for $\omega=0.5$ in the $\mathbf{b} \tau$-space. 
$\Sigma^{3}$ is obtained from $\Sigma^{6}$ through letting $\kappa=\mu=0$ and substituting the variables $\Theta$ and $\mathbf{w}$ in terms of $u \equiv(\mathbf{b}, \tau) \in \mathbf{R}^{2} \times(0, \infty)$.

As long as $\mathbf{c} \neq 0, \Sigma^{3}$ has up to four rest points, numbered as $u_{0}, u_{1}, u_{2}, u_{3}$ (according to the dimension of their stable manifolds). For thermodynamical reasons only viscous profiles $u_{i} \rightarrow u_{j}$ for shock waves with $u^{-}=u_{i}$ and $u^{+}=u_{j}$ which satisfy $i<j$ can exist. Certain combinations $\left(u_{i}, u_{j}\right)$ among these - namely those for which $(i, j) \in\{0,1\} \times\{2,3\}$-give rise to nonclassical or "intermediate" shock waves.

Depending on the ratio of $\nu$ and $\lambda$ the dynamical system $\Sigma^{3}$ undergoes a global heteroclinic bifurcation. Regarding the existence of viscous profiles and this bifurcation it can be proven (cf. [12, 8] and references therein):

Theorem 5.1. There exists $\omega^{*}=\omega^{*}(\bar{q}, \lambda, \nu)>0$ such that we have for

$$
\begin{aligned}
& \omega \equiv \frac{\nu}{\lambda}<\omega^{*} \quad: \quad u_{0} \rightarrow u_{1}, u_{2} \rightarrow u_{3} \text { exist } \\
& \left.\omega=\omega^{*} \quad: \quad \begin{array}{l}
u_{0} \rightarrow u_{1}, u_{2} \rightarrow u_{3}, \\
u_{1} \rightarrow u_{2}
\end{array}\right\} \text { exist, } \\
& \left.\omega>\omega^{*} \quad: \quad \begin{array}{l}
u_{0} \rightarrow u_{1}, u_{2} \rightarrow u_{3}, \\
u_{1} \rightarrow u_{2}, u_{0} \rightarrow u_{2}, \\
u_{1} \rightarrow u_{3}, u_{0} \rightarrow u_{3}
\end{array}\right\} \text { exist. }
\end{aligned}
$$

In all three cases no other orbits exist.

Now we present numerical results for all possible types of viscous profiles in MHD. For $\bar{q}$ we have chosen

$$
\bar{q}=(1.0,0.15,0.0,1.8,1.0) .
$$

$\omega^{*}$ then can be calculated numerically using special properties of the MHD-system 7]. We obtain $\omega^{*} \approx 0.019$.

First we consider an example where $\omega$ equals $0.5>\omega^{*}$. In Figure 6 orbits of almost all types of heteroclinic connections that exist due to Theorem 5.1 are displayed. There is a pair of orbits connecting the rest points $u_{1}$ and $u_{2}$. Heteroclinic manifolds of dimension 2 relate $u_{0}$ to $u_{2}$ and $u_{1}$ to $u_{3}$. The profiles $u_{0} \rightarrow u_{1}$ and $u_{2} \rightarrow u_{3}$ correspond to classical Laxian shock waves. All these orbits form the boundary of a three-dimensional heteroclinic manifold connecting $u_{0}$ with $u_{3}$ (not displayed).
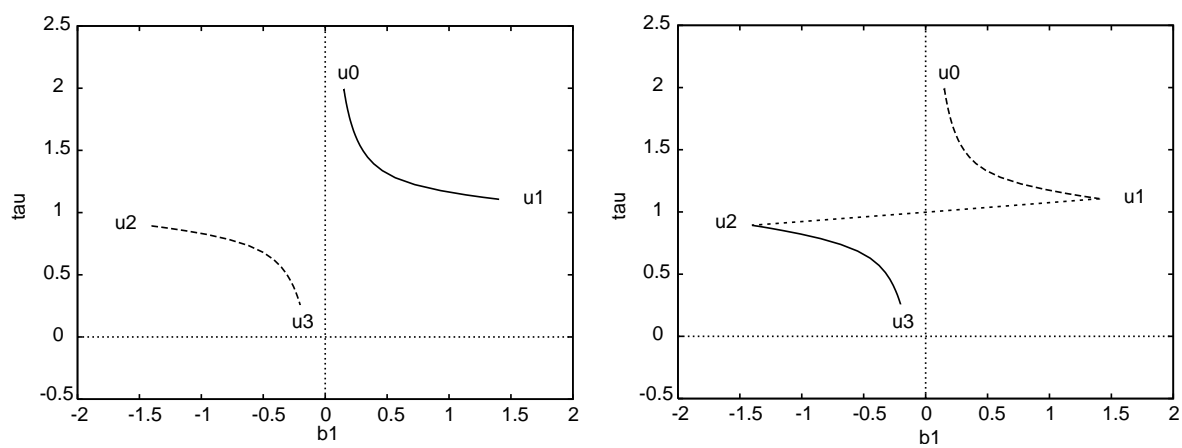

FiguRE 7. Viscous profiles for $\omega=0.01$ and $\omega=\omega^{*}$ in the $b^{1} \tau$-plane. 

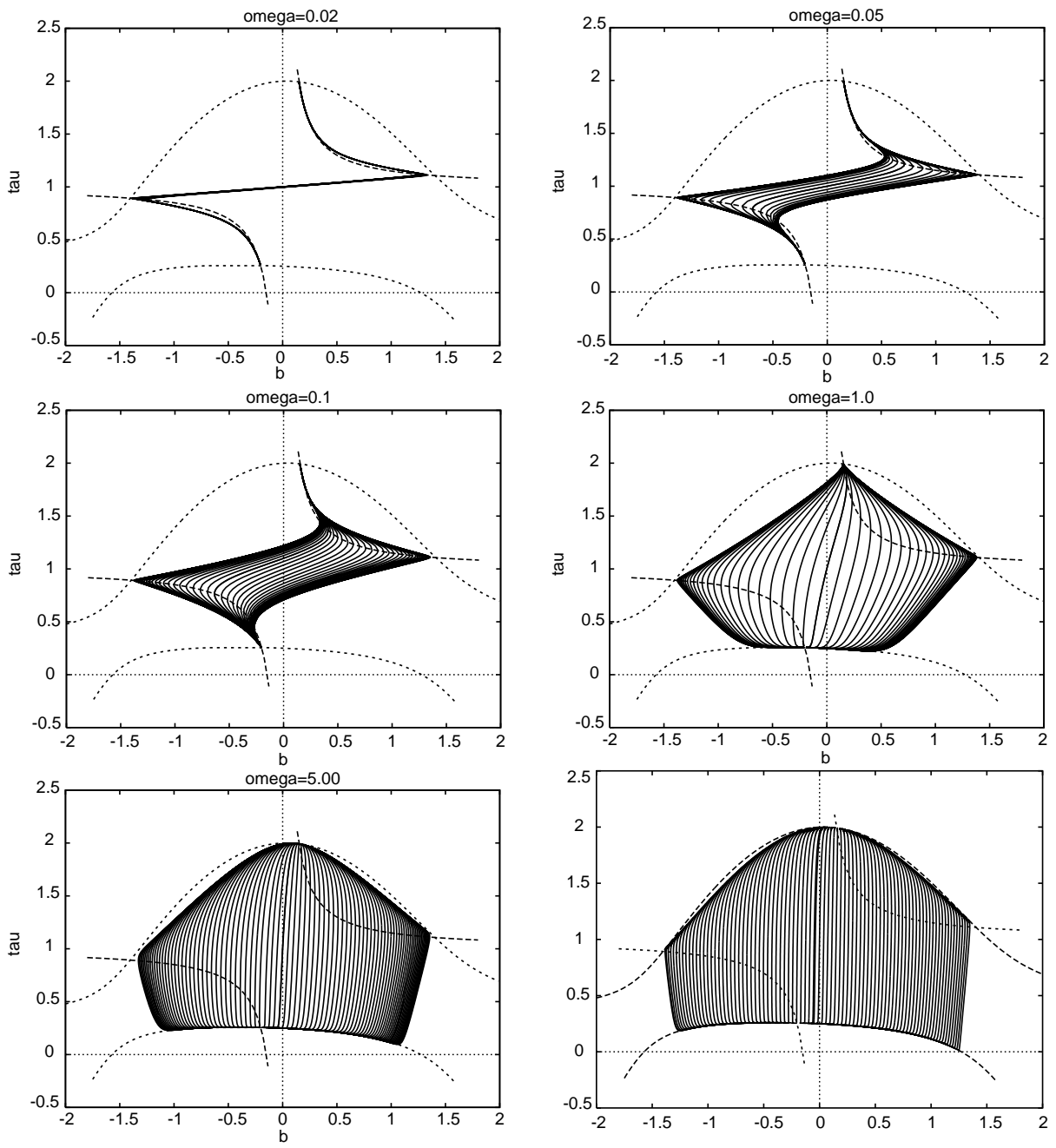

Figure 8 . Orbits connecting $u_{0}$ and $u_{3}$ in the $b^{1} \tau$-plane.

We proceed with a parameter study for the ratio $\omega$. For this sake we note that in our case the $\left(b^{1} \tau\right)$-plane constitutes an invariant manifold for $\Sigma^{3}$, the orbits $u_{0} \rightarrow$ $u_{1}, u_{2} \rightarrow u_{3}$ belong to this plane for all $\omega, u_{1} \rightarrow u_{2}$ for $\omega=\omega^{*}$ (cf. [8]). Consequently the bifurcation scenario presented in Theorem [5.1] can be completely described in the plane. Figure 7 displays the orbits $u_{0} \rightarrow u_{1}, u_{2} \rightarrow u_{3}$ for $\omega=0.01<\omega^{*}$ and additionally the orbit $u_{1} \rightarrow u_{2}$ for $\omega=\omega^{*}$. Figure 8 shows orbits in the $\left(b^{1}, \tau\right)$-plane which belong to the three-dimensional heteroclinic manifold connecting $u_{0}$ and $u_{3}$ for

$$
\omega=0.02,0.05,0.1,1.0,5.0,15.0 .
$$

Also the nullclines of the flux of $\Sigma^{3}$ in the $b^{1} \tau$-plane are displayed. 


\section{REFERENCES}

[1] G. Bader and U. Ascher, A new basis implementation for a mixed order boundary value ODE solver, SIAM J. Sci. Stat. Comput. 8, 483-500 (1987). MR 88f:65118

[2] F. Bai, A. Spence and A. M. Stuart, The numerical computation of heteroclinic connections in systems of gradient partial differential equations, SIAM J. Num. Anal., 53/3, 743-769 (1993). MR 94h:65107

[3] W.--J. Beyn, The numerical computation of connecting orbits in dynamical systems, IMA J. Numer. Anal., 9, 379-405 (1990). MR 91i:65146

[4] F. R. DeHoog and R. Weiss, An approximation theory for boundary value problems on infinite intervals, Computing, 24, 227-239 (1980). MR 82f:65087

[5] E. J. Doedel and M. J. Friedman, Computation and continuation of invariant manifolds, SIAM J. Numer. Anal., 28, 789-808 (1991). MR 92e:34058

[6] H. Freistühler and T.-P. Liu, Nonlinear stability of overcompressive shock waves in a rotationally invariant system of viscous conservation laws, Commun. Math. Phys. 153, No.1, 147-158 (1993). MR 94f:35084

[7] H. Freistühler and C. Rohde, A numerical study of existence and bifurcation of MHD shock profiles, in preparation.

[8] H. Freistühler and P. Szmolyan, Existence and bifurcation of viscous profiles for all intermediate magnetohydrodynamic shock waves, SIAM J. Math. Anal., 26, No.1, 112-128 (1995). MR 95j:35183

[9] H. Freistühler and K. Zumbrun, Examples of unstable viscous shock waves, preprint.

[10] C. Fries, Nonlinear asymptotic stability of general small-amplitude viscous Laxian shock waves, J. Differ. Equations 146, 185-202 (1998). MR 99h:35132

[11] D. Gilbarg, The existence and limit behaviour of the one-dimensional shock layer, Amer. J. Math., 73, 1-13 (1951). MR 13:401e

[12] A. G. Kulikovskij and G. A. Lyubimov, On the structure of an inclined magnetohydrodynamic shock wave (English. Russian original), J. Appl. Math. Mech. 25, 171-179 (1961);

[13] M. Lentini and H. B. Keller, Boundary value problems over semi-infinite intervals and their numerical solution, SIAM J. Numer. Anal., 17, 577-604 (1980). MR 81j:65092

[14] T.-P. Liu, Nonlinear stability of shock waves for viscous conservation laws, Am. Math. Soc. Mem. 328, Providence, AMS (1985). MR 87a:35127

[15] P. Markowich, A theory for the approximation of solutions of boundary value problems on infinite intervals, SIAM J.Math. Anal., 13, 484-513 (1982). MR 83e:34024

[16] G. Moore, Computation and parametrization of connecting orbits, IMA J. Numer. Anal., 15, 245-264 (1995). MR 96a:34087

[17] K. J. Palmer, Exponential dichotomies and transversal homoclinic points, J. Differ. Equations, 55, 225-256 (1984). MR 86d:58088

[18] A. Szepessy and Z. Xin, Nonlinear stability of viscous shock waves, Arch. Ration. Mech. Anal. 122, No.1, 53-103 (1993). MR 93m:35125

[19] D. Serre, Systèmes de lois de conservation I, Paris (1996). MR 99b:35139

[20] G. Vainikko, Funktionalanalysis der Diskretisierungsmethoden, Leipzig (1976). MR 57:7997

[21] C.C. Wu, Formation, structure, and stability of MHD intermediate shocks, J. Geophys. Res. 95, 8149-8175 (1990).

Max-Planck-Institut für Mathematik in Den Naturwissenschaften, Inselstr. 22-26, D-04103 Leipzig, Germany

E-mail address: hfreist@mis.mpg.de

Institut für Angewandte Mathematik, Albert-Ludwigs-Universtät Freiburg, Hermann-Herder-Str. 10, D-79104 Freiburg, Germany

E-mail address: chris@mathematik.uni-freiburg.de 